\title{
Fast Single Layer Cylindrical and Helicoidal Coil With Voids Between Turns Electromagnetic Field Calculation to Be Used in Superconducting Current Limiter Simulator for Design Purpose
}

\author{
C. A. Baldan, R. C. Freitas, R. P. Homrich, D. G. Pinatti, E. Ruppert Filho, and C. Y. Shigue
}

\begin{abstract}
This paper presents a mathematical method to quickly calculate the flux density vector $B$ produced by the current circulating in a resistive single-phase superconducting electrical current limiter (RSCL), at any point of its whole space, using the Biot-Savart's Law. As the calculation using the exact form of the turns of each coil (single layer cylindrical coil with helicoidal form presenting voids (free space) between neighbor turns) is computationally very cumbersome to be used in a RSCL dynamic simulator, it is proposed in this paper to substitute the real coil, only for the subject of the calculation, by an imaginary coil formed by plane closed circular turns with the same number of turns, the same height and radius as the real coil. Each turn of the imaginary coil is placed exactly in the medium position between successive turns of the actual coil and will carry the same current. The coil self-inductance is also calculated to verify the accuracy of the proposed method.
\end{abstract}

Index Terms-Current limiter, electromagnetics, finite elements, superconductor.

\section{INTRODUCTION}

$\mathbf{E}$ LECTRICAL power system loads are expanding continuously and the short circuit levels at its different points are increasing so that the capacity of the electrical protection devices are exceeded. To solve this problem it is necessary to implement fast short-circuit electrical protection features which do not interfere in the regular operation of the system and that are self-restored after the short-circuit occurrence to guarantee the electrical power system stability.

The resistive superconducting electrical current limiter (RSCL) is an effective solution to solve this problem [1], [2] because it presents all that mentioned desired characteristics to perform as a very fast switch introducing an electrical resistance in series with the circuit in less than $1 \mathrm{~ms}$ limiting the short-circuit current to a required value.

Manuscript received October 21, 2003. This work was supported by CPFLPaulista under Grant 4600000767.

C. A. Baldan, D. G. Pinatti, and C. Y. Shigue are with the Chemical Engineering Faculty of Lorena (FAENQUIL), Brazil (e-mail: cabaldan@ demar faenquil.br)

R. C. Freitas and E. R. Filho are with the Systems and Energy Control Department, Computer and Electrical Engineering School, Campinas University (DSCE/FEEC/UNICAMP), Campinas 13081970, Brazil (e-mail: ruppert@fee.unicamp.br)

R. P. Homrich is with the Electrical Engineering Department, Federal University of Rio Grande do Sul (DEE/UFRGS), Rio Grande do Sul State, Brazil (e-mail: homrich@eletro.ufrgs.br).

Digital Object Identifier 10.1109/TASC.2004.830287

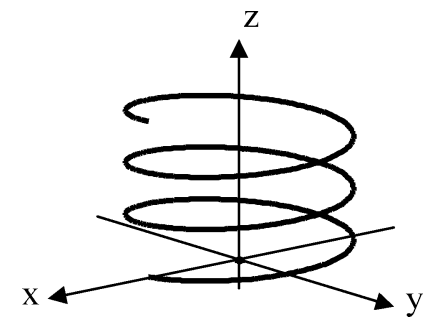

Fig. 1. Helicoidal coil with voids between turns.

When the electrical current quickly increases due to an overload or short-circuit occurrence exceeding a critical current $\left(\mathrm{I}_{\mathrm{c}}\right)$ at a given temperature of the cryogenic coolant fluid, the superconducting material transits from the superconducting state to the conducting state (quench) due to the increase of the magnitude of the magnetic flux density vector (B) on the material surface above the critical magnetic field $\mathrm{Bc}$.

The RSCL are constituted by single-layer concentric cylindrical coils with nonmagnetic core like fiber glass [3]. Due to the electrical and coolant characteristics those coils present helicoidal form and voids between neighbor turns as shown in Fig. 1.

As described in [3], the magnetic field generated by the current circulating on each turn of the RSCL coils contributes to the increase of the magnitude of the vector B on the superconducting material surface.

The largest value uses to occur near the medium position of each coil, making this point a source of the quench propagation.

The calculation of the vector B on the surface of the superconducting material in that mentioned region is of fundamental importance in the design phase of the RSCL coils because this value is used in the mathematical simulation of the dynamic behavior of the RSCL so as to adapt to the design values to permit the correct actuation of the limiter just when submitted to high currents and so that the quench doesn't occur at rated electrical current.

The dynamic simulator used in the design of the RSCL, as detailed described in [3], is based on the critical current of the superconducting material in function of the magnetic induction in its surface $\left(I_{c} \times B\right)$ at a given cryogenic fluid temperature as well as in the self and mutual inductances of the several coils constituting the RSCL. 


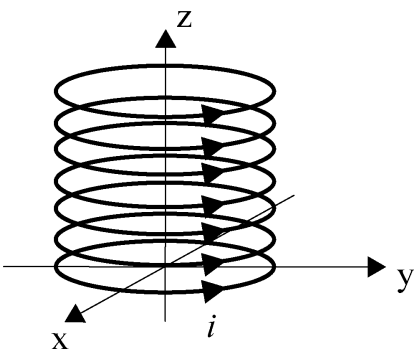

Fig. 2. The plane closed circular turns coil.

A RSCL, constituted of concentric coils, can be mathematically represented by a set of differential equations describing the electrical circuits representing each coil. Each one of those electrical circuits comprises the coil electrical resistance (in the normal conducting state), the self inductance of the coil and all the mutual inductances between coils.

The RSCL must be designed so that the inductances before mentioned result in a minimum value of the equivalent inductance of the RSCL so that it presents a very low impedance to the electrical power system when in the superconducting state.

At each step of the numerical solution of the set of differential equations [3], it is necessary to calculate the current circulating in the coils and the magnetic flux density vector at each point of the superconducting material. The material characteristic curve $\left(\mathrm{I}_{\mathrm{c}} \times \mathrm{B}\right)$ is verified to check if the material is in a quench condition, that is, if for the $\mathrm{B}$ calculated value the electrical current value is above the $\left(I_{c} \times B\right)$ curve.

With the correct design of the coil parameters of the RSCL it has the guarantee that the quench will occur only at the overcurrent desired value and that it will not occur during the regular operation of the electrical power system.

The most important calculation involved in the RSCL design is the calculation of the vector B at each point of the coils. It makes possible to evaluate the inductances and then to simulate the dynamic behavior of the RSCL.

In [4] it was presented a mathematical method using the BiotSavart's law to evaluate the magnetic flux density at each point of the RSCL coils considering the exact form of the turns that is helicoidal with voids between turns. This type of vector B calculation, named Helicoidal Method, is very accurate but it is also very high time consumption method.

In this paper it is presented an alternative method to the above mentioned method where the helicoidal coil with voids between turns are substituted by coils constituted by plane closed circular turns uniformly spaced along the nonmagnetic core carrying the same current as shown in Fig. 2.

The Biot-Savart's law is also used and it is the best solution to calculate the vector B considering that the RSCL simulator used in the design step requires the calculation of the vector $\mathrm{B}$ at any place of the RSCL at each step of the differential equation numerical solution. It could be thought to use a finite element software to do this job but to do that it would be necessary to have the possibility to solve the set of differential equations together with the finite element equations which would be troublesome and not usual in finite element commercial software package.

All the calculations shown were numerically implemented in this paper through a specific software developed for this project

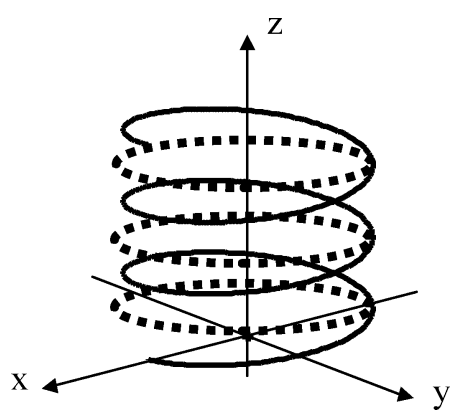

Fig. 3. Plane turns at the average position.

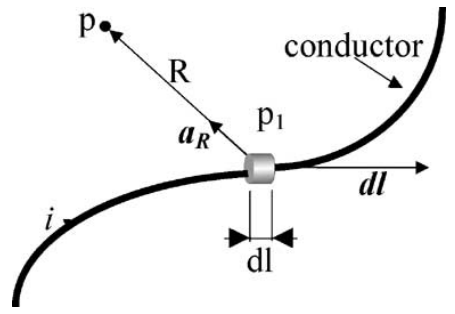

Fig. 4. Illustration of the Biot-Savart's law.

and a low temperature RSCL using CuNi matrix superconductor with 3 concentric coils parallel connected was designed, built and tested successfully as reported in [3]. At this time a new RSCL using high temperature superconductor ceramic material are being designed to be built and tested.

\section{The Coil With Plane Closed Circular Turns}

The turns of the coil shown in the Fig. 1 are substituted by plane closed circular turns, as shown in Fig. 2, placed at the average position between two successive turns of the coil in Fig. 1 as shown in Fig. 3.

The calculation of the vector B at any point of the RSCL coils can be done taking a differential current element of the coil as shown in Fig. 4 and applying the Biot-Savart's law in its differential form, as shown in (1), to calculate the differential of the vector B:

$$
\mathrm{d} \vec{B}=\frac{\mu_{0} i}{4 \pi} \frac{\mathrm{d} \vec{l} \times \vec{a}_{\mathrm{r}}}{|\vec{R}|^{2}} .
$$

\section{Magnetic FluX Density Vector Calculation}

According to the Biot-Savart's law the vector B at any point of the RSCL space, produced by the electrical current circulating in the coil can be determined as shown in Fig. 4. Applying the Biot-Savart's law to the plane closed circular turn, the vector B can be calculated at any distance $\mathrm{R}$ from the turn carrying the electrical current $i$ as shown in Fig. 5, through the calculation of the integral of (1) along the curve described by the turn.

Using the cartesian coordinate system the vector B can be well determined, at any point of the space, by the components $\mathrm{B}_{\mathrm{x}}, \mathrm{B}_{\mathrm{y}}$ and $\mathrm{B}_{\mathrm{z}}$ as shown in (2)to (6).

$$
\begin{aligned}
d \vec{l} & =r d \theta \vec{a}_{\theta} \\
\vec{a}_{\theta} & =-\operatorname{sen} \theta \mathrm{i}+\cos \theta \mathrm{j} \\
d \vec{l} & =(-r \operatorname{sen} \theta \mathrm{i}+r \cos \theta \mathrm{j}) d \theta
\end{aligned}
$$




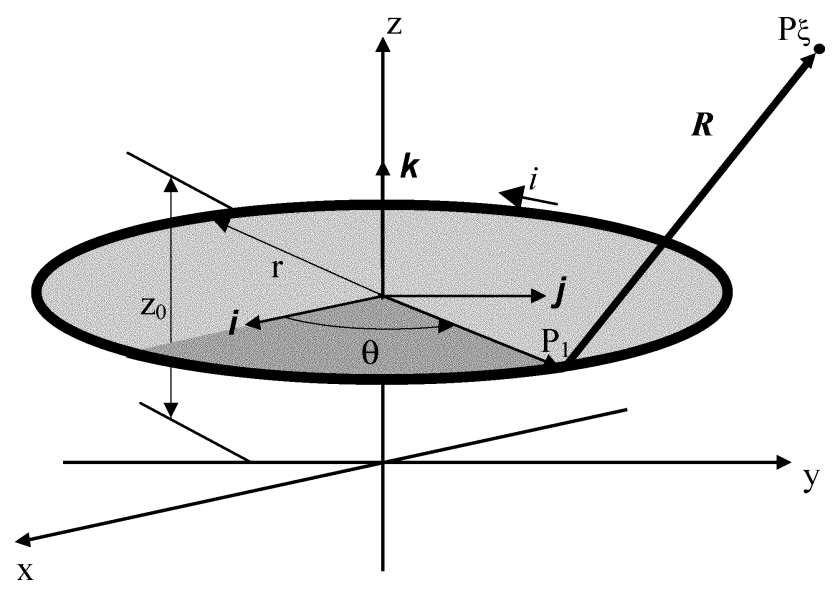

Fig. 5. Biot-Savart's law applied to a plane closed circular turn.

$$
\vec{R}=\left(x_{\xi}-r \cos \theta\right) \mathrm{i}+\left(y_{\xi}-r \operatorname{sen} \theta\right) \mathrm{j}+\left(z_{\xi}-z_{0}\right) \mathrm{k}
$$

(See equations (5) to (6) at bottom of page)

\section{Calculation of the SElF AND Mutual Inductances OF THE RSCL}

The vector B calculation method shown above is also necessary to calculate the self inductance of each coil and also the mutual inductances between coils of the RSCL. As the coils present non magnetic cores the system can be considered magnetically linear and those inductances may be calculated using (7). In this equation $\lambda$ is the linkage magnetic flux linking any coil and $i$ is the current circulating in this coil (for self-inductance calculation) or is the current in any other coil (for mutual-inductance calculation).

$$
\mathrm{L}=\frac{\lambda}{i}
$$

The magnetic flux linking each turn can be determined by the (8). In this equation the vector B is calculated considering the proper electrical current as mentioned in the paragraph before

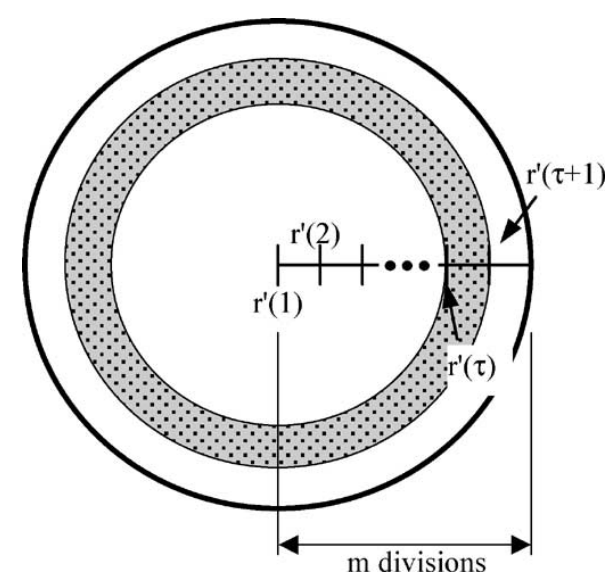

Fig. 6. Discretization of the radius of the coil.

and the vector $\mathrm{dA}$ is the area vector element of the considered turn surface.

$$
\phi=\int_{A} B_{z} d A
$$

The surface integral (8) can be numerically calculated dividing the surface area of each plane closed circular turn in $\mathrm{m}$ concentric rings as shown in Fig. 6. The magnetic flux linking each ring is approximated by (9) where the area of the ring $A_{\tau}$ is given by the (10). This approximation is as good as the number $\mathrm{m}$ of rings increases.

$$
\begin{aligned}
\overline{\phi(\tau)} & =\frac{B_{z}(\tau)+B_{z}(\tau+1)}{2} \cdot A_{\tau} \\
A_{\tau} & =\pi\left[r^{\prime 2}(\tau+1)-r^{2}(\tau)\right]
\end{aligned}
$$

The total magnetic flux linking the turn $\mathrm{k}$ of the coil is given by (11) and the linkage flux of the coil having $\mathrm{N}$ turns is given by (12).

$$
\phi_{k}=\sum_{\tau=1}^{m} \overline{\phi(\tau)}
$$

$$
\begin{aligned}
\frac{\vec{a}_{R}}{|\vec{R}|^{2}} & =\frac{\left(x_{\xi}-r \cos \theta\right) \mathrm{i}+\left(y_{\xi}-r \operatorname{sen} \theta\right) \mathrm{j}+\left(z_{\xi}-z_{0}\right) \mathrm{k}}{\left[x_{\xi}^{2}+y_{\xi}^{2}+r^{2}-2 r\left(x_{\xi} \cos \theta+y_{\xi} \operatorname{sen} \theta\right)+\left(z_{\xi}-z_{0}\right)^{2}\right]^{\frac{3}{2}}} \\
B(\mathrm{x}, \mathrm{y}, \mathrm{z}) & =\mathrm{B}_{\mathrm{x}} i+\mathrm{B}_{\mathrm{y}} j+\mathrm{B}_{\mathrm{z}} k \\
B_{x} & =\frac{\mu_{0} i}{4 \pi} \int_{0}^{2 \pi} \frac{r \cos \theta\left(z_{\xi}-z_{0}\right)}{\left[x_{\xi}^{2}+y_{\xi}^{2}+r^{2}-2 r\left(x_{\xi} \cos \theta+y_{\xi} \operatorname{sen} \theta\right)+\left(z_{\xi}-z_{0}\right)^{2}\right]^{\frac{3}{2}}} d \theta \\
B_{y} & =\frac{\mu_{0} i}{4 \pi} \int_{0}^{2 \pi} \frac{r^{2}-r\left(x_{\xi} \cos \theta\left(z_{\xi}-z_{0}\right)\right.}{\left[x_{\xi}^{2}+y_{\xi}^{2}+r^{2}-2 r\left(x_{\xi} \cos \theta+y_{\xi} \operatorname{sen} \theta\right)+\left(z_{\xi}-z_{0}\right)^{2}\right]^{\frac{3}{2}}} d \theta \\
B_{z} & =\frac{\mu_{0} i}{4 \pi} \int_{0}^{2 \pi} \frac{\left[x_{\xi}^{2}+y_{\xi}^{2}+r^{2}-2 r\left(x_{\xi} \cos \theta+y_{\xi} \operatorname{sen} \theta\right)+\left(z_{\xi}-z_{0}\right)^{2}\right]^{\frac{3}{2}}}{[\theta}
\end{aligned}
$$


TABLE I

RSCL COILS DATA

\begin{tabular}{lccc}
\hline \hline & Coil a & Coil b & Coil c \\
\cline { 2 - 4 } Number of turns (N) & 86 & 74 & 66 \\
Internal radius (mm) & 67.0 & 77.5 & 87.5 \\
Coil length (mm) & 258.0 & 111.0 & 264.0 \\
Void between turns (mm) & 2.494 & 0.9993 & 3.492 \\
Helicoidal method & & & \\
Self inductance (mH) & 0.4254 & 0.7295 & 0.3996 \\
CPU time (h) & $11: 29$ & $10: 24$ & $11: 34$ \\
This work & & & \\
Self inductance (mH) & 0.4189 & 0.7183 & 0.3926 \\
CPU time (h) & $1: 51$ & $1: 22$ & $1: 05$ \\
Self inductance relative error (\%) & 1.53 & 1.54 & 1.75 \\
CPU time ratio & 6.21 & 7.61 & 10.68 \\
\hline \hline
\end{tabular}

TABLE II

COPPER WIRE COILS DATA

\begin{tabular}{lccc}
\hline \hline & Coil a & Coil b & Coil c \\
\cline { 2 - 4 } Number of turns (N) & 5 & 10 & 20 \\
Internal radius (mm) & 150.0 & 150.0 & 150.0 \\
Coil length (mm) & 37.5 & 75.0 & 150.0 \\
Void between turns (mm) & 7.0 & 7.0 & 7.0 \\
Measured inductance (mH) & 0.0165 & 0.0480 & 0.1337 \\
Helicoidal method & & & \\
Self inductance (mH) & 0.0169 & 0.0488 & 0.1354 \\
CPU time (min) & 25 & 81 & 340 \\
Relative error vs measured (\%) & 2.61 & 1.53 & 1.26 \\
This work & & & \\
Self inductance (mH) & 0.0159 & 0.0468 & 0.1312 \\
CPU time (min) & 0.38 & 1.48 & 6.0 \\
Relative error vs measured (\%) & 3.50 & 2.59 & 1.91 \\
CPU time ratio & 65.79 & 54.73 & 56.67 \\
\hline \hline
\end{tabular}

$$
\lambda=\sum_{k=1}^{\mathrm{N}} \phi_{k}
$$

\section{RESULTS}

The self-inductances of a set of 6 different coils were used to validate the proposed method of the magnetic flux density vector calculation. It was used the 3 coils of the built RSCL mentioned in the item I of this paper which data are given in Table I and also a set of 3 different coils made of copper wire which data are given in the Table II. In the first case (Table I) the self-inductances of the coils were calculated by the proposed method and by the before mentioned Helicoidal Method [4] and the results are compared concerning the self inductance relative errors and also CPU time consumed for each calculation. In the second case (Table II) the self-inductance of each coil were measured using a GenRad 1659 RLC Digibridge and calculated using the Helicoidal Method. The results were compared concerning also the relative errors and CPU time consumed for each calculation.

To compare the CPU time consumed in this method with the CPU time consumed in the helicoidal method, both computer programs were run in the same computer and the run time was measured from the start to stop the run.

As it can be seen in Tables I and II the self-inductance values calculated using this proposed method presents a very small errors when compared with the calculated by the helicoidal method and with the measured values.

The CPU time was significantly reduced by more than 5 times thus confirming that this method is reliable and computationally efficient for the purpose of RSCL design.

\section{CONCLUSION}

A fast mathematical method to calculate the magnetic flux density vector in a RSCL using a substitution of an helicoidal coil by a hypothetical plane closed circular turns parallel mounted coil was presented. The accuracy is very close to that found in [4] and the CPU time consumption to perform the calculation is very low. This way this method of calculation can be used in the design of resistive superconducting electrical current limiters without loss of the complete accuracy of the limiter operation condition.

\section{REFERENCES}

[1] Y. Wu and Y. Yin, "Fault-current limiter applications in medium and high-voltage power distribution systems," IEEE Trans. Industry Applications, vol. 34, pp. 236-242, Jan/Feb. 1998.

[2] P. C. Michael, K. Ryu, and O. Tsukamoto, "Quench currents of a C. superconductor in supercritical helium," IEEE Trans. Appl. Supercond., vol. 5, no. 2, pp. 373-376, Jun. 1995.

[3] R. P. Homrich, E. R. Filho, D. G. Pinatti, C. A. Baldan, and C. Y. Shigue, "Single-phase resistive superconductor electrical current limiter," IEEE Trans. Appl. Supercond., vol. 12, no. 1, pp. 1386-1389, March 2001.

[4] C. A. Baldan, R. C. Freitas, R. P. Homrich, D. G. Pinatti, E. R. Filho, and C. Y. Shigue, "Single Layer Cylindrical and Helicoidal Coil With Voids Between Successive Turns Electromagnetic Field Calculation to be Used in Superconductor Current Limiter Simulator for Design Purpose,", this conference. 